\title{
The Fate of a Total Talar Allograft to Treat Traumatic Talar Enucleation, Fifteen Years after Transplantation in Teenage Patients
}

\author{
Reynders Piet ${ }^{1, *}$ and Dobre Cristina, ${ }^{2, *}$ \\ ${ }^{I}$ Department of Orthopedics and Traumatology, University Hospitals Brussels, Campus Brugmann, Arthur Van \\ Gehuchtenplein 4, 1020 Brussels, Belgium \\ ${ }^{2}$ Department of Rehabilitation, University Hospitals Brussels, campus Brugmann, Arthur Van Gehuchtenplein 4, 1020 \\ Brussels, Belgium
}

\begin{abstract}
The authors describe two cases of post-traumatic loss of the whole talus treated with a total talar allograft with a follow-up of fifteen years.

In one case sizing of the graft was difficult, necessitating the resection of a middle segment of the talar dome. In this case after eight years the talar dome collapsed in height for one third. In the second case where we didn't have these problems of sizing, survival of the graft after fifteen years was seen. In both cases active revascularization as shown with bone scintigraphy was still going on after this time. Although both patients have a stable ambulatory hindfoot, the functional outcome was poor in both cases.
\end{abstract}

Keywords: Talar fractures, enucleation of the talus, fresh osteochondral allografts, Hindfoot fractures.

\section{INTRODUCTION}

Total traumatic dislocation of the talus from all its surrounding joints (tibiotalar, subtalar and talonavicular) is an extremely rare injury, involving $2 \%$ of all talar injuries $[1,2]$. The management is controversial. When the talus can be retrieved, re-implantation seems to be valuable option whenever adequate debridement of the hindfoot can be obtained [3]. In other case, tibiocalcaneal arthrodesis is the only alternative. In selected cases, Boyd's hindfoot arthrodesis can achieve a stable plantigrade foot for limited community ambulation with relatively few complications [4].

We describe two cases with total talar loss in one case and total talar loss together with the loss of the os cuneiforme intermedium and laterale and os cuboideum. The written informed consent from each subject was obtained.

\section{CASE REPORTS}

Case 1: A nineteen years old female had a motorcycle accident, she sustained an isolated talectomy (Fig. 1a,b). The talus could not be retrieved because of heavy contamination. After serial debridement, the hindfoot was

*Address correspondence to these authors at the Department of Rehabilitation, University Hospitals Brussels, campus Brugmann, Arthur Van Gehuchten-plein 4, 1020 Brussels, Belgium;

E-mail: Pieter.reynders@chu-brugmann.be and

Department of Rehabilitation, University Hospitals Brussels, campus Brugmann, Arthur Van Gehuchtenplein 4, 1020 Brussels, Belgium;

Tel: +32473361420; E-mail: Cristina_dobre79@yahoo.com placed in a spanning external fixator, with gradual distraction of the calcaneo-crural space (Fig. 2a, b). To prevent ingrowth of scar tissue, the distraction zone was packed with gentamycin beads. The patient received a combination of cefazolin 1 gram intravenously two times daily and Gentamycine intravenously with a serum concentration of $5 \mu \mathrm{g} / \mathrm{ml}$ for three days. After the soft tissue healed, a fresh talar graft, from a female donor of the same age who died off a non-traumatic intra-cranial bleeding, was transplanted.

Because the talus was too large, a middle segment of the talar dome needed to be removed (Fig. 3a, b).

Also, the absences of the lateral ankle ligaments necessitated the reconstruction of the lateral ankle ligaments using the peroneus brevis tendon using a modification of the technique described by Chrisman and Snook. The modification consisted in making a passage through the lateral facet of the talus and anterior part of the calcaneum (Fig. 4a). The deltoid ligame.nt on the medial side of the ankle was reconstructed with a part of the tibialis posterior tendon (Fig. 4b). The traumatic wound was left open and skin grafted when the granulation tissue formed.

This patient ambulated for one year with a patellar tendon bearing apparatus after which she used custom made shoes.

At four years, gradual collapse of the proximal third of the talus was observed. The degree of collapse remained constant from that time until the last control fifteen years after the accident (Fig. 5 a, b) and (Fig. 6a,b,c,d). 


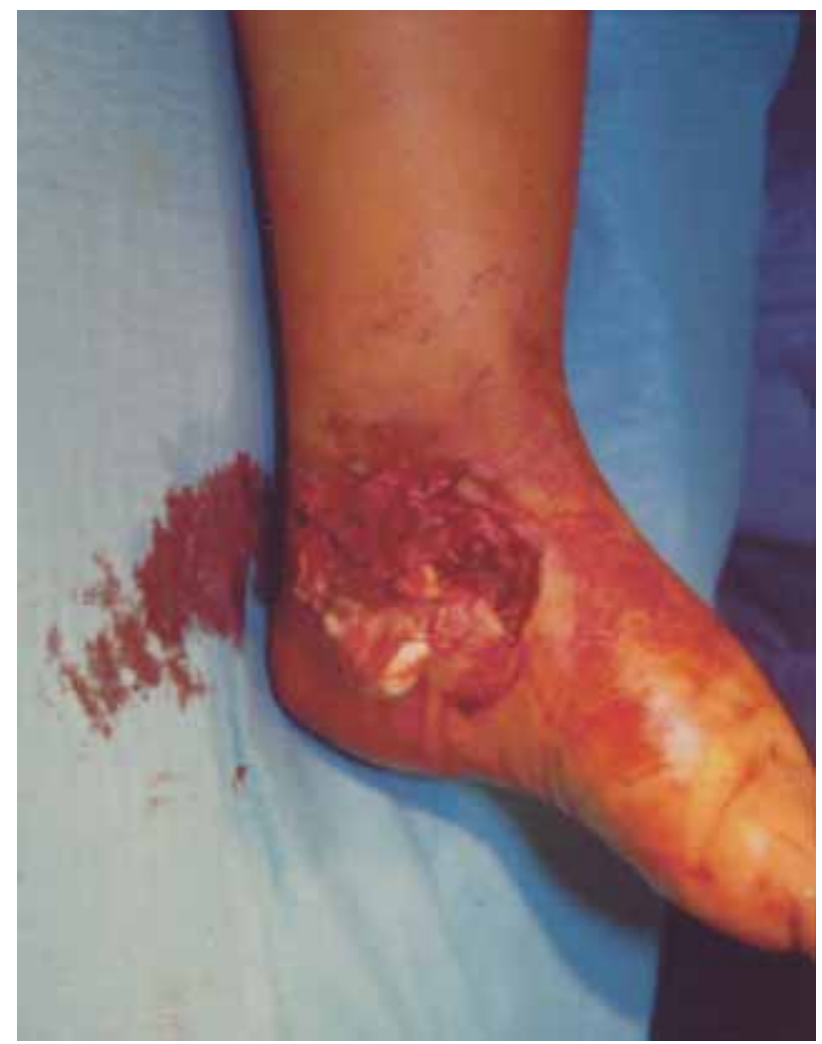

Fig. (1a). View of the medial laceration after complete extrusion of the talus.

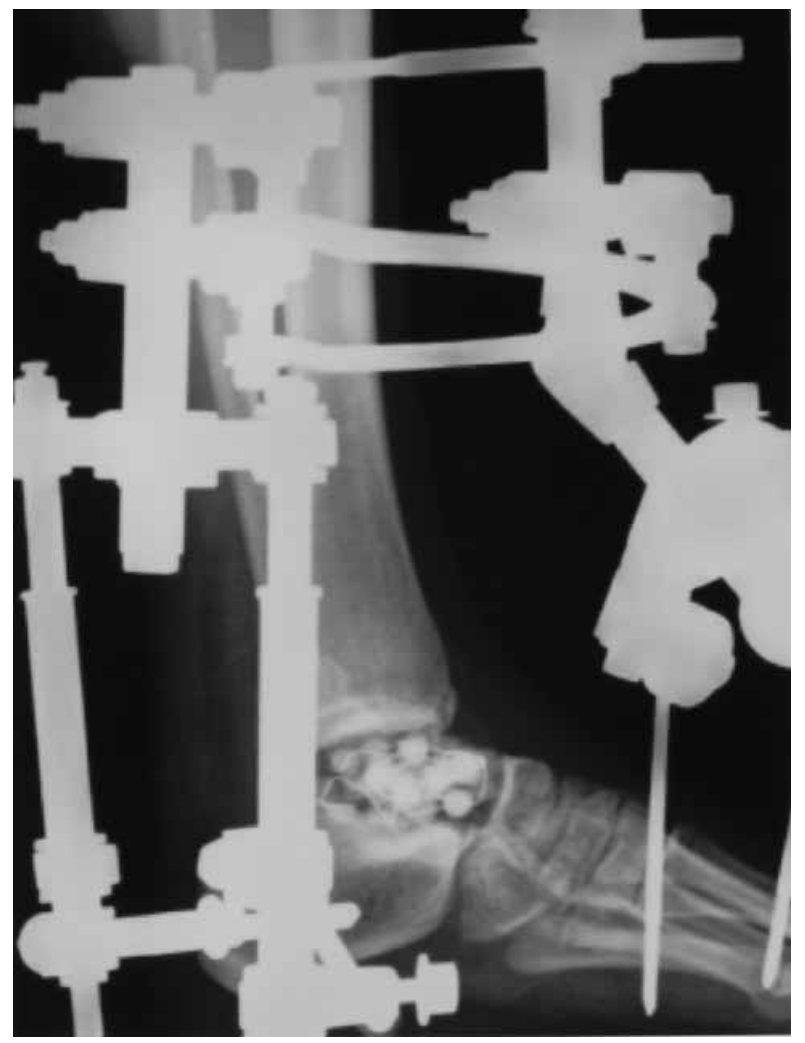

Fig. (1b). Radiograph after placement of the external fixator. The space between the tibia and calcaneus is filled with gentamycin beads. 


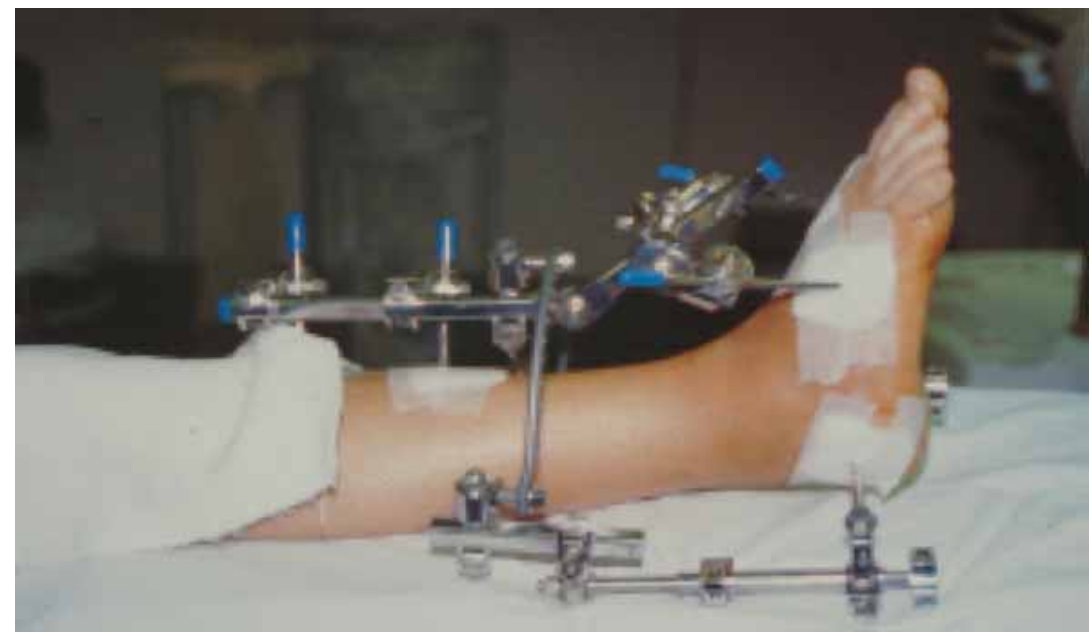

Fig. (2a). Lateral view of the external fixator.

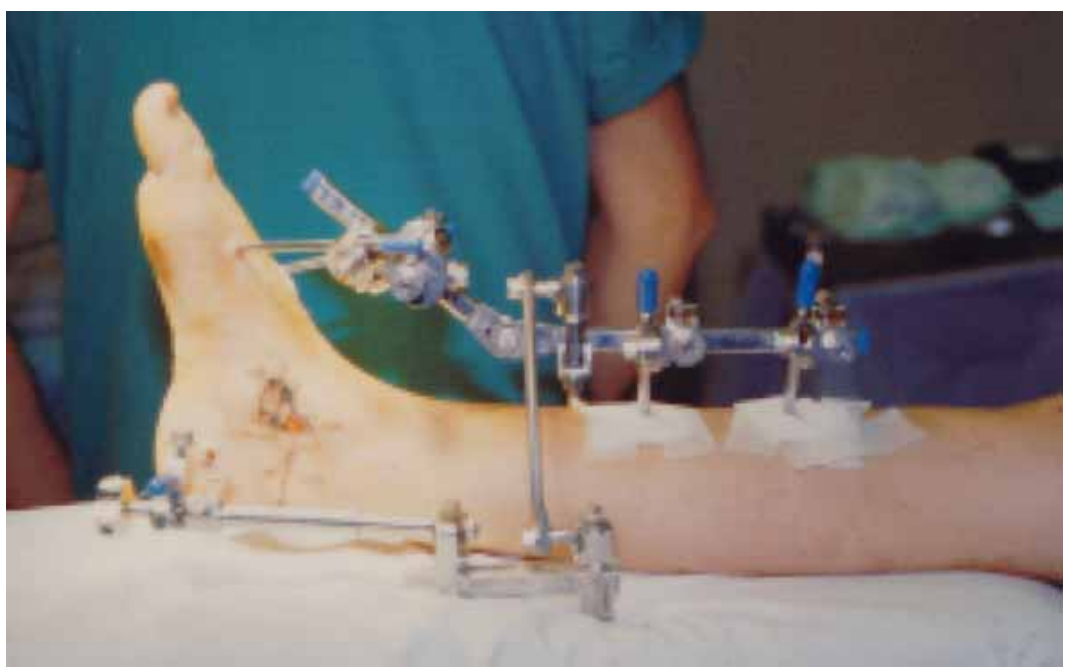

Fig. (2b). Medial view of the external fiaxtor.

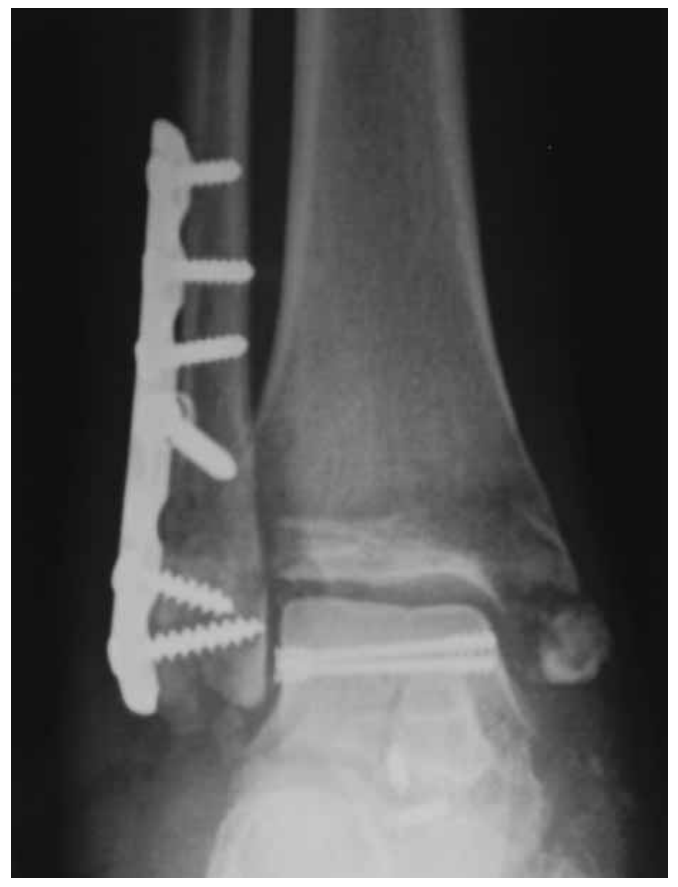

Fig. (3a). Radiograph after transplantation of the talus allograft. 


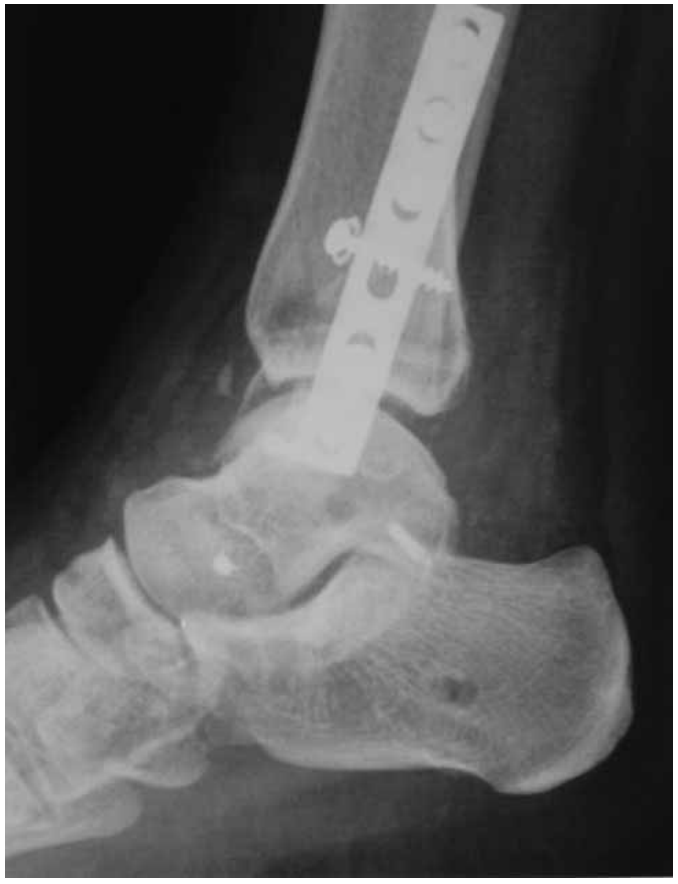

Fig. (3b). Lateral radiographic image after transplantation of the talus.

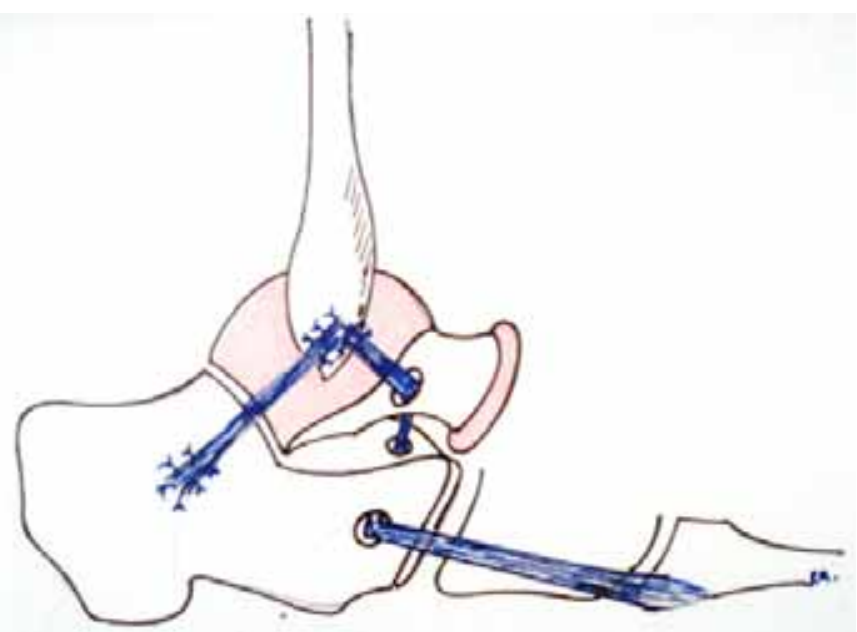

Fig. (4a). Draft of the reconstruction of ligametum deltoideum.

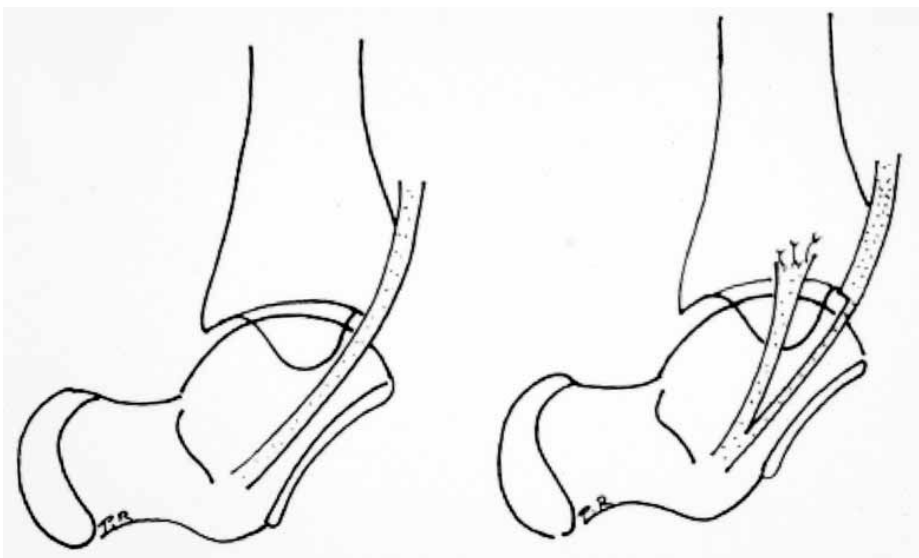

Fig. (4b). Scheme of the reconstruction of the lateral ankle band with a strip of the peroneus brevis tendon. 


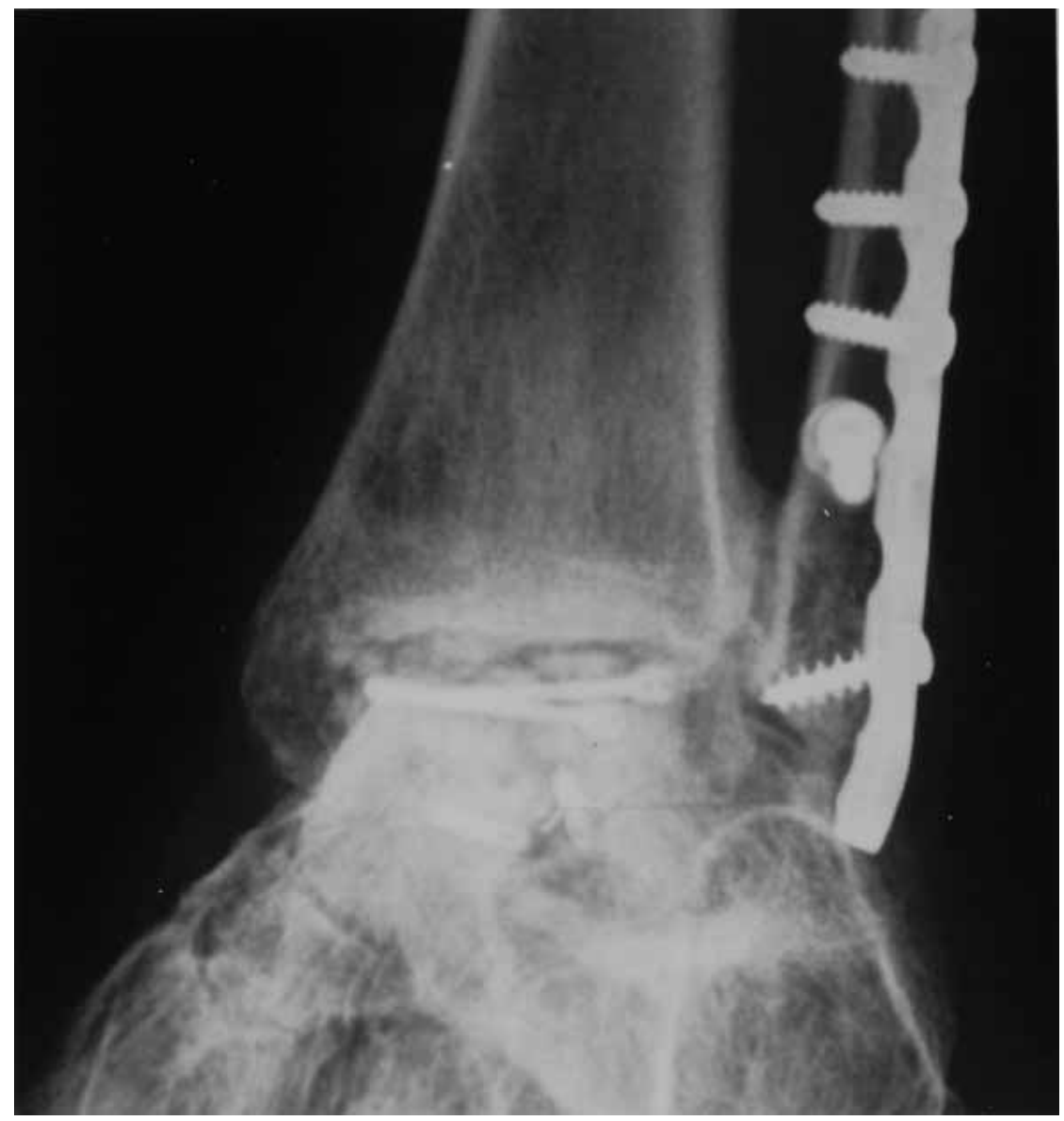

Fig. (5a). Radiograph fifteen years after talus transplantation with partial collapse of the talar dome.

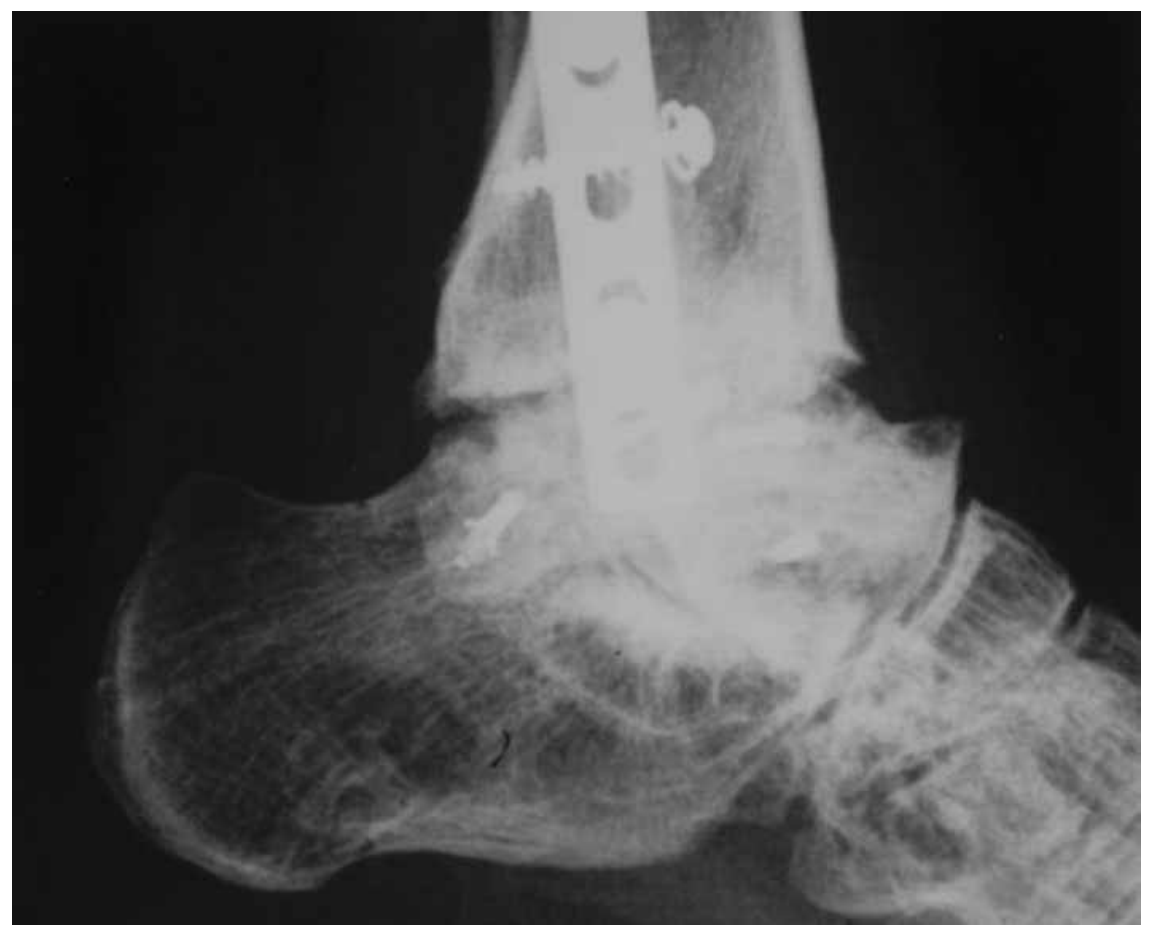

Fig. (5b). Lateral view of the same ankle, depicting the flattening of the talo crural space. 


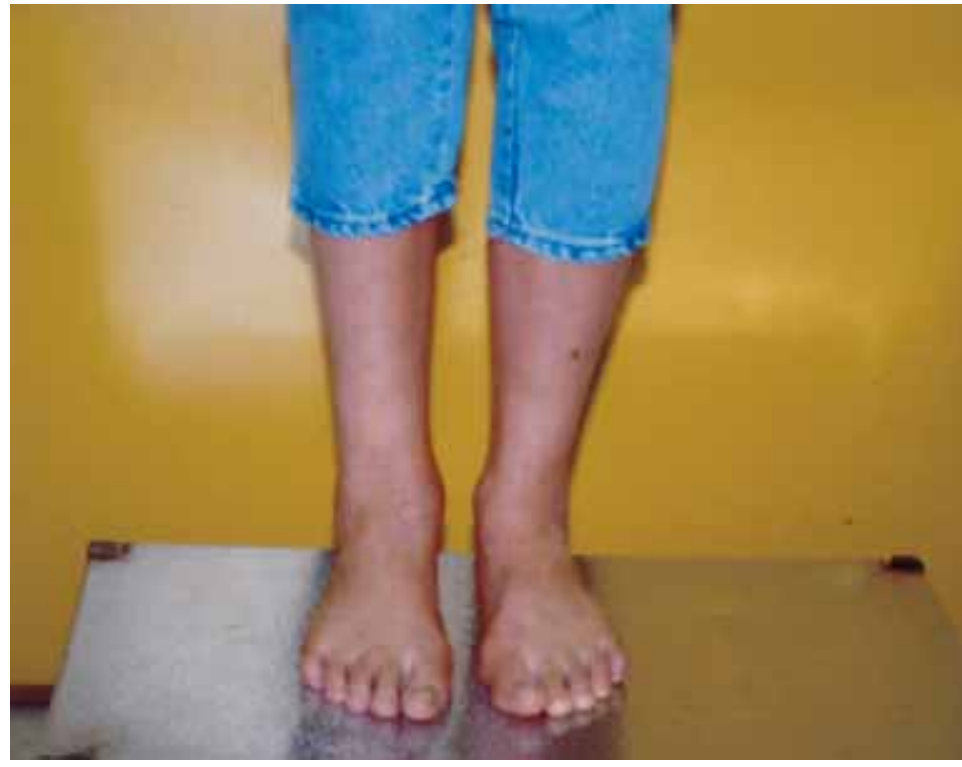

a

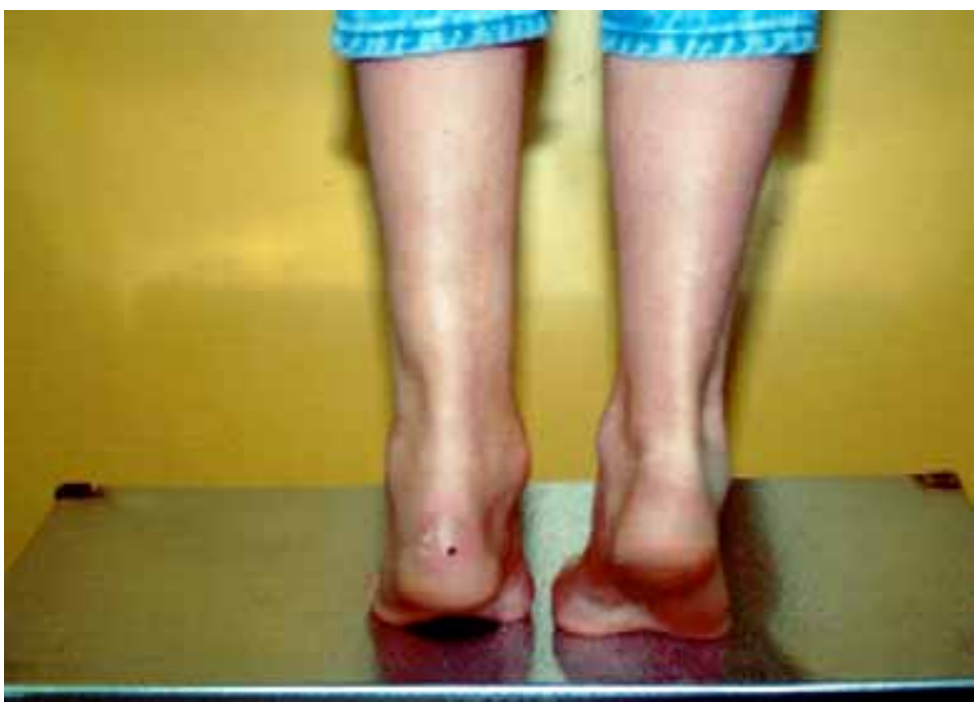

b

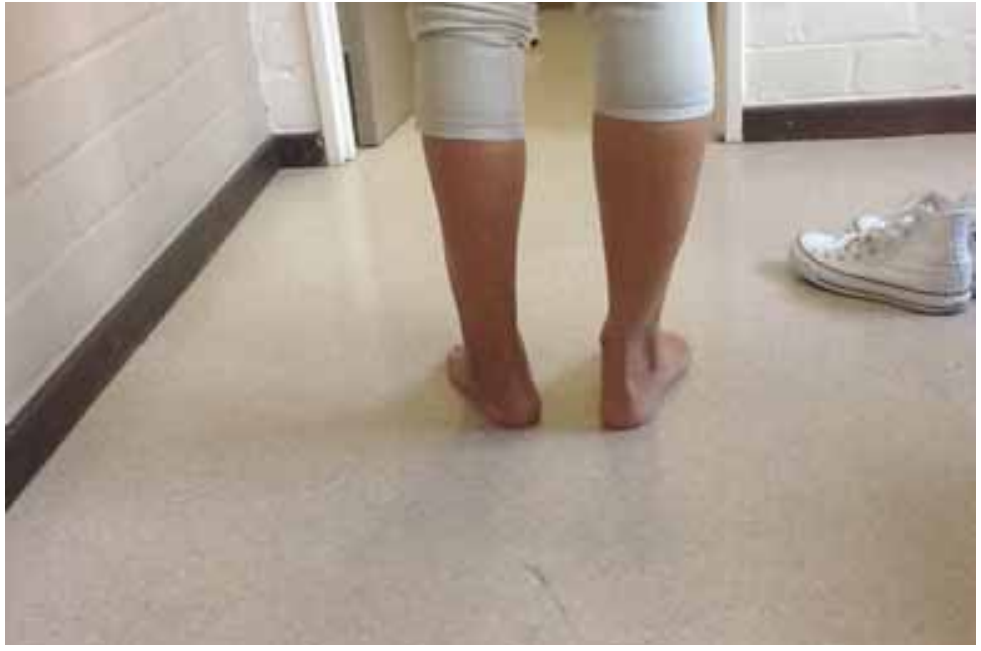

c 


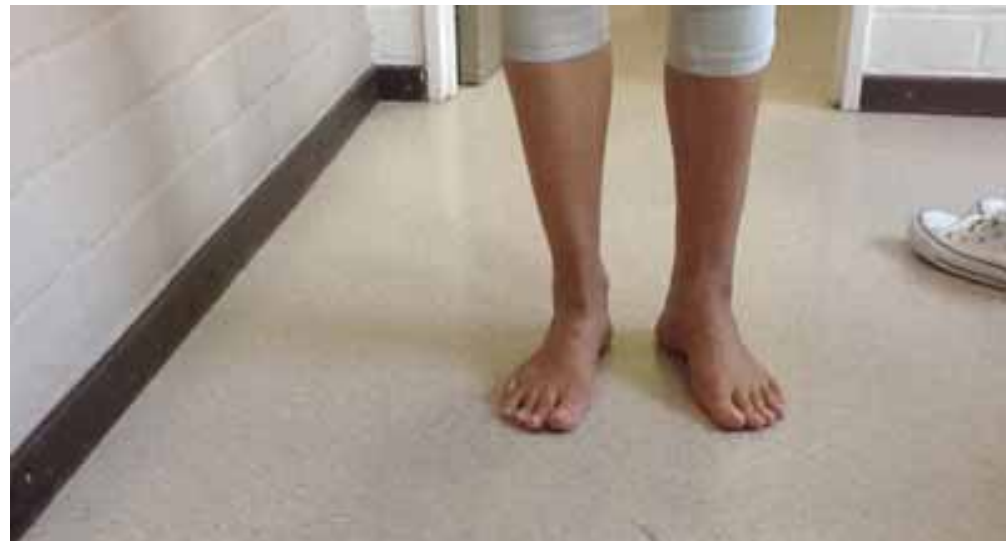

d

Fig. (6). (a, b, c, d) Clinical picture of case $\mathrm{n}^{\circ}$ 1. After eight years a,b and after fifteen years c,d .Muscle waisting of the calf muscles and slight widening of the hindfoot and varus deformity at fifteen years post-incident.

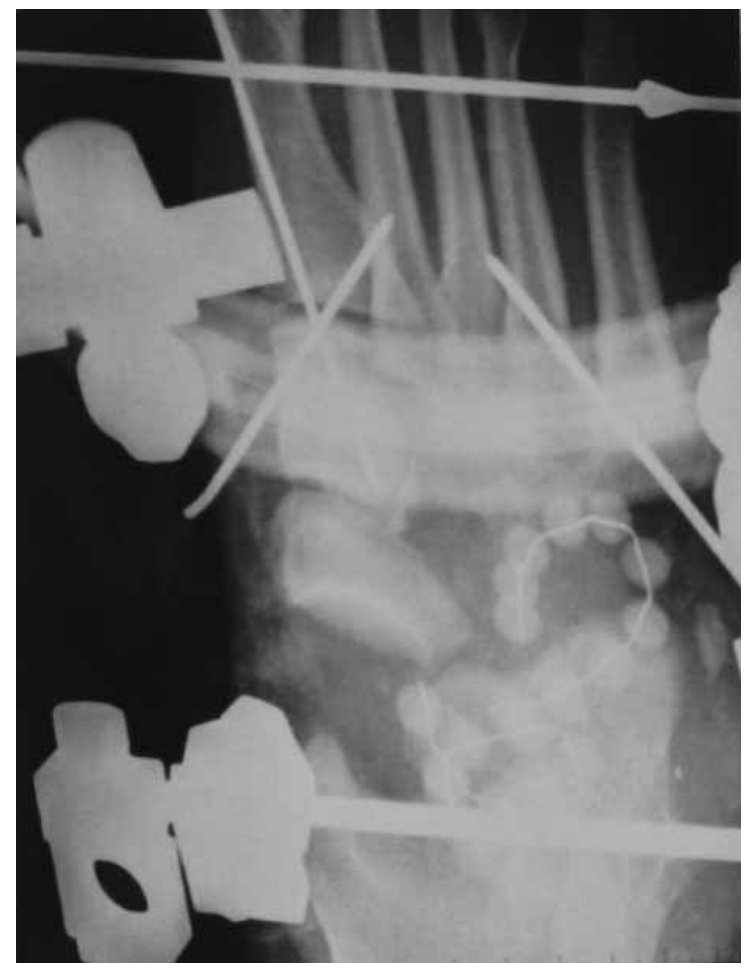

Fig. (7). Radiograph of the foot \& ankle showing the complete loss of the talus, the two cuneiform bones and the cuboid. The empty space is filled up with gentamycin beads.

In this case bone scanning ( ${ }^{99 \mathrm{M}} \mathrm{TC}-\mathrm{MDP}$ scintimetry) of the talus after six years showed vascularity of the graft (Fig. 10).

This patient is working as a primary school teacher without restrictions. Although she has some days of less discomfort, most of the time she is in pain. She has no complains of instability of her ankle. She walks with a slight external rotation of her foot to accommodate for the lack of tibio-pedal movement. She can't participate in sports activities. Tibio-pedal passive movement is $25^{\circ}$ with her ankle in $4^{\circ}$ of varus (Fig. 6a, b,c,d). The AOFAS hindfoot score was $<50$ at one and two years, 55 at three years, 65 at eight years and 55 at fifteen years (AOFAS, ankle-hindfoot score; 100points; excellent 90-100, good 75-89, failure < 50)
Subjective patient evaluation was categorized as very satisfied, satisfied, uncertain and dissatisfied. This patient felt uncertain about her result. Subjectively, she can cope with her situation and refused several times additional surgery.

\section{CASE 2}

A seventeen years old female had a car crash. Her car collided into the rear of another car. She had an isolated injury to her left foot with an open wound on the lateral side of the ankle and foot. Extrusion of the talus, cuboid and lateral and intermediate cuneiform were noticed (Fig. 7). Throughout serial debridement was performed and a 


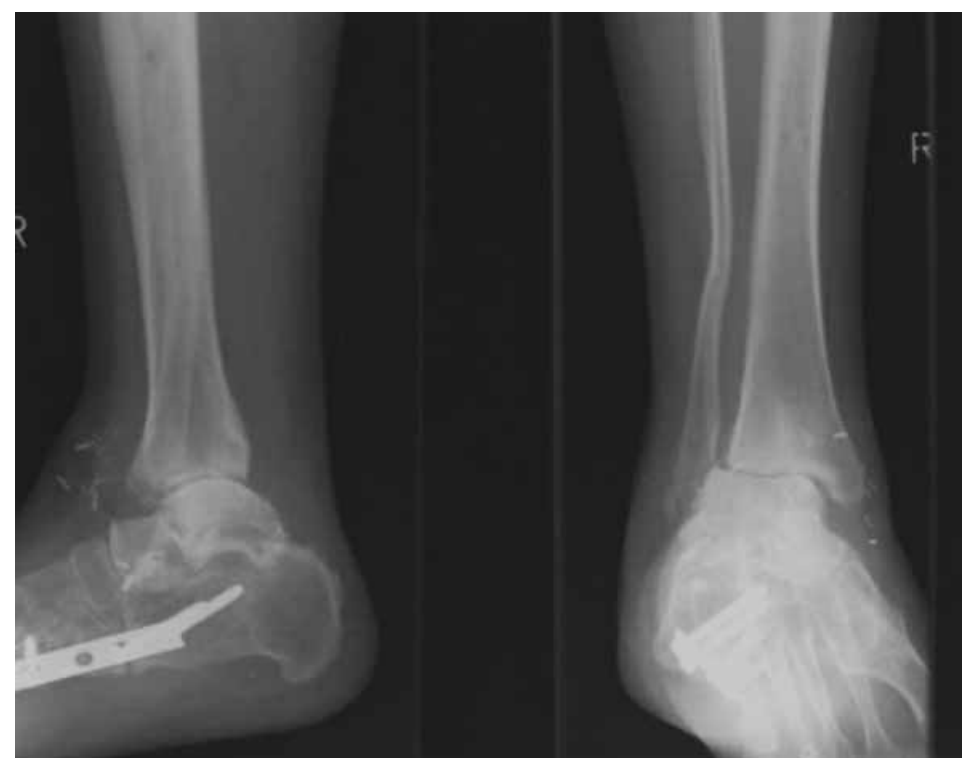

a

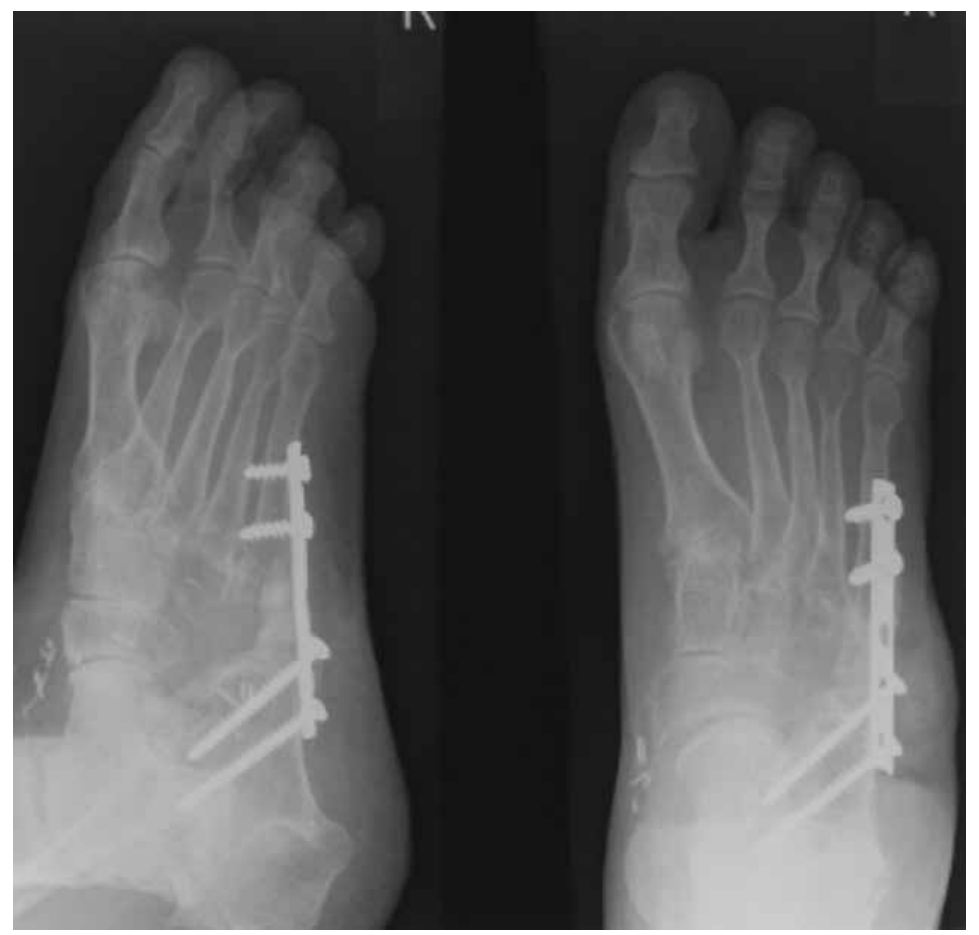

b

Fig. (8). (a) and (b) Radiographs fifteen years after transplantation. Slight narrowing of the joint space and instability of the ankle joint. Reconstruction of the fifth ray of the foot with a tricortical graft and plate osteosynthesis.

spanning external fixator under traction was applied, bridging the hind and forefoot. The cavity between the pilon and the os calcis was filled with gentamycin beads. After five days, a free flap (latissimus dorsi flap) was used to cover the defect. Three months after the incident a fresh total talar bone transplant was performed. Due to sufficient bony stability, no additional ligamentous reconstructions of the hindfoot were necessary. At the same time a plate osteosynthesis was performed stabilizing the fifth ray of the foot. The gap in the midfoot was filled with an tricortical autologous bone graft. In total, this patient needed seven surgical interventions to deal with her situation.
The hybrid external fixator stayed on for an additional six weeks after which the patient started walking with a patellar tendon bearing apparatus for another four months. Since then she used custom made orthopaedic shoes for ambulation. After two years, gradual narrowing of the ankle joint line was visible on standard radiographies. This narrowing of the ankle joint stabilized since that time until her last follow-up (Fig. 8a, b) and (Fig. 9a,b,c,d). At her latest follow-up, fifteen years after trauma, active tibio-pedal movement of $40^{\circ}$ is possible, but no active subtalar movement was possible. Passive tibiopedal movement of $60^{\circ}$ was observed together with $20^{\circ}$ of subtalar movement. During gait, no real 

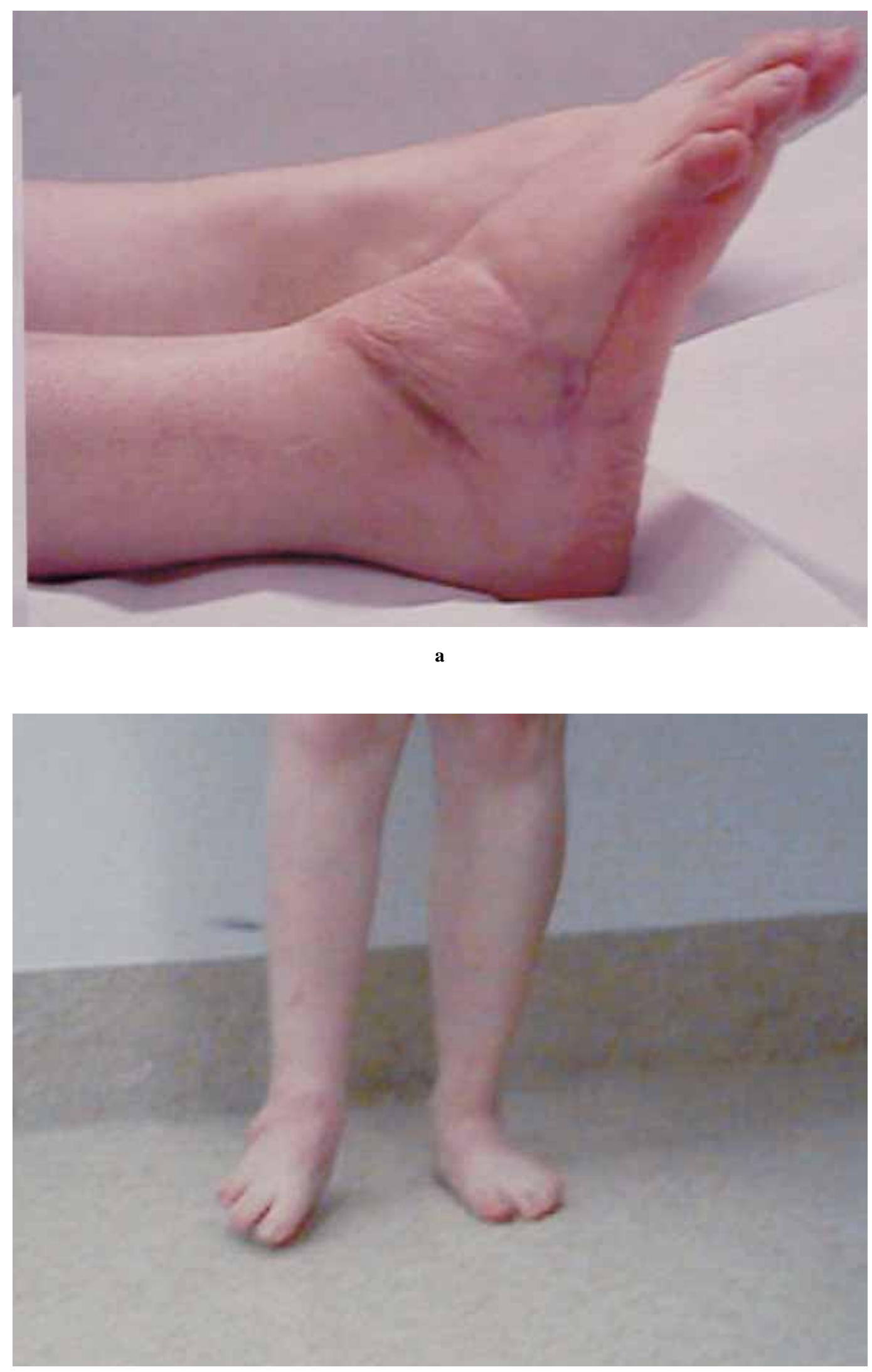


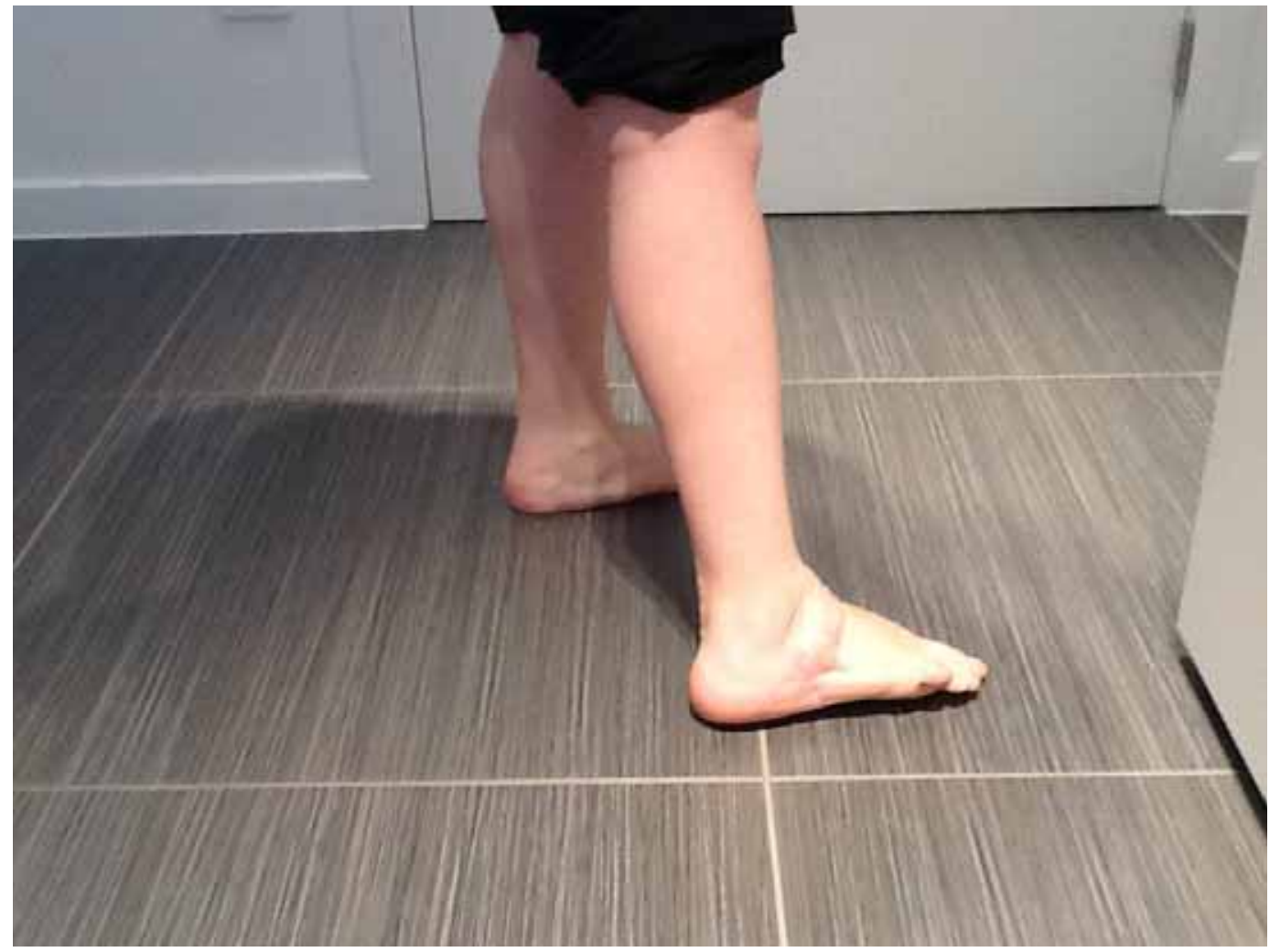

c

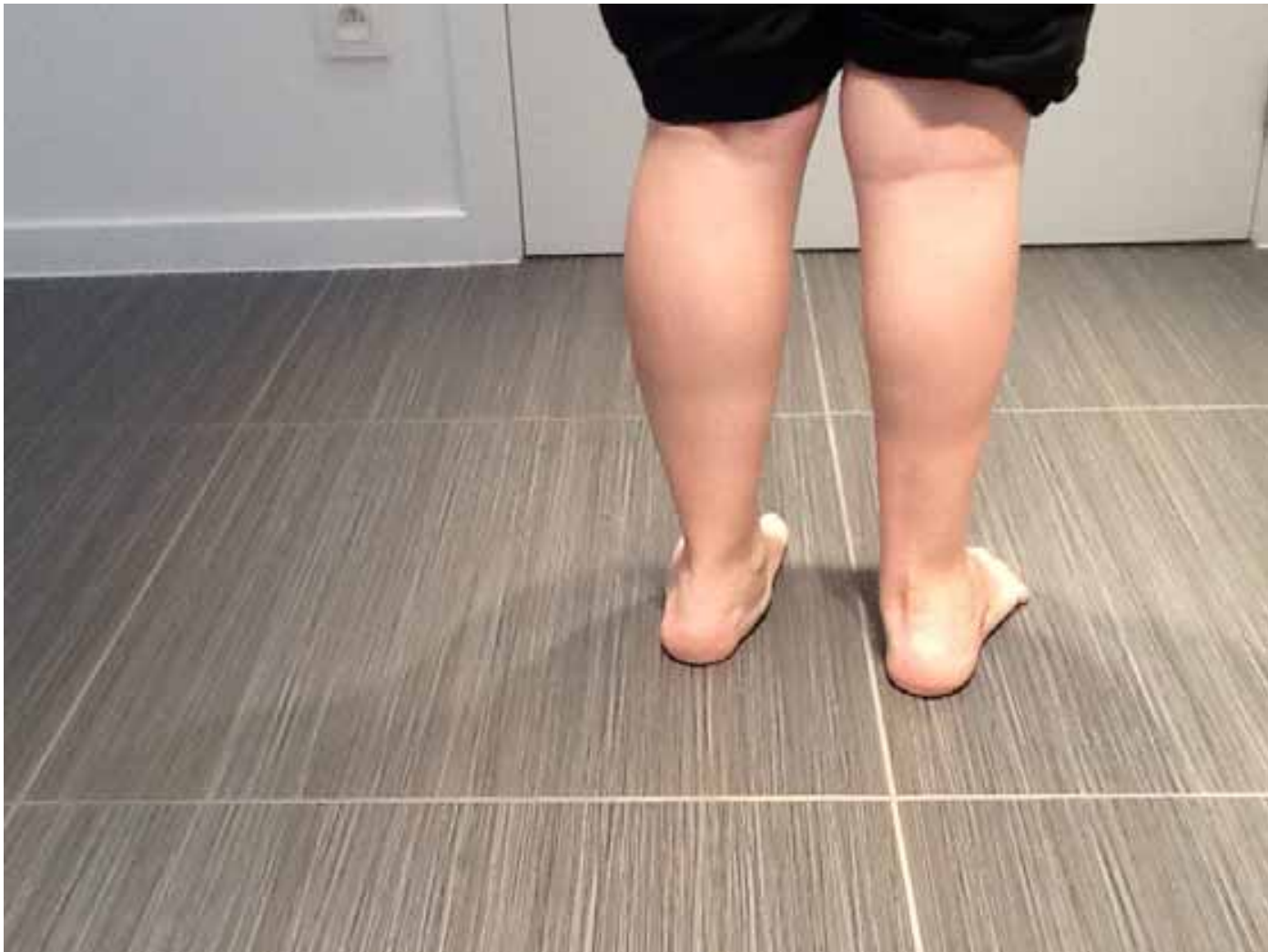




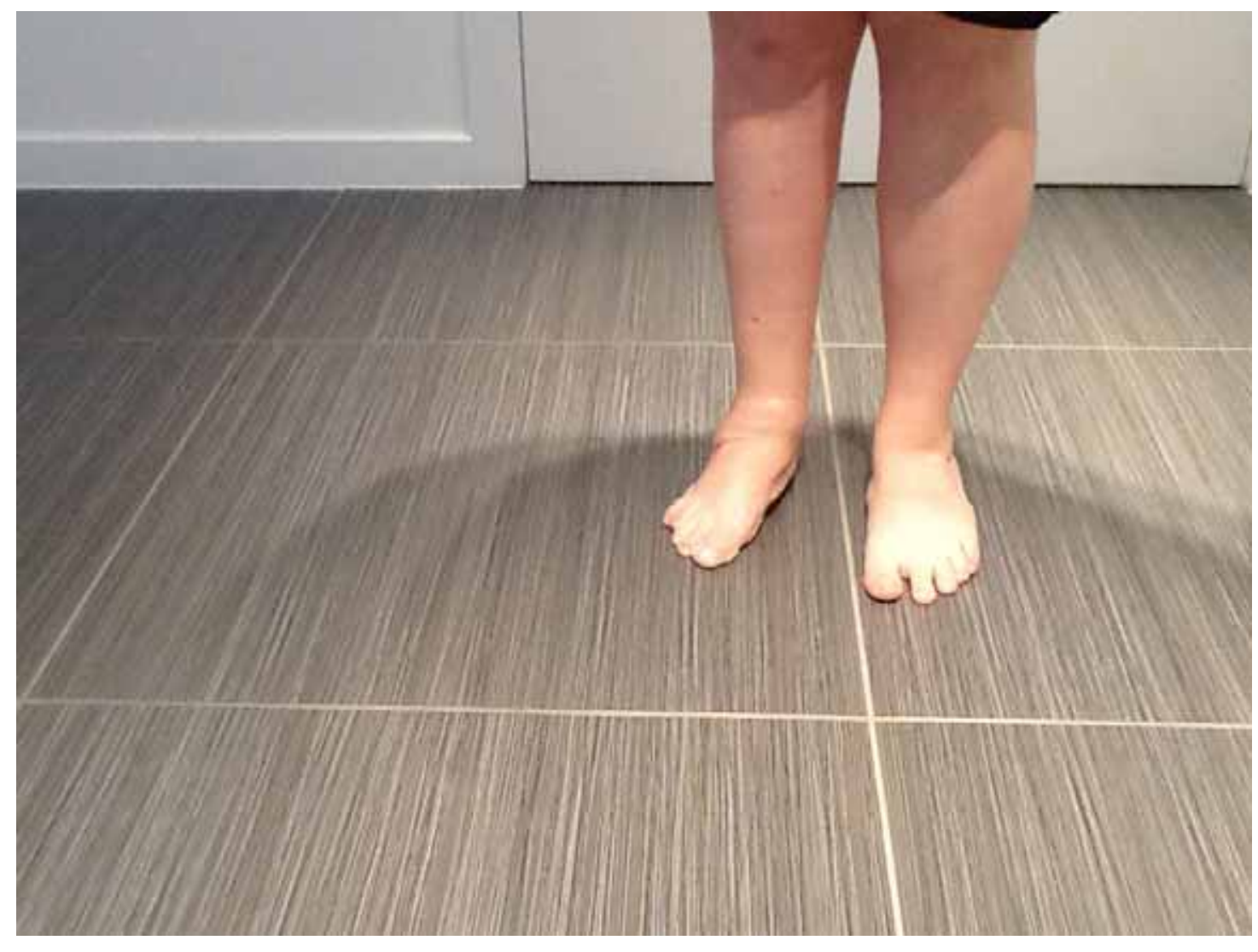

e

Fig. (9). (a , b, c, d, e) Clinical results after eight years a\&b and after fifteen years c,d,e. Limited ankle movement. Only a jog of movement at the subtalar joint.

ankle motion was visible. She has a constant discomfort on walking, improves at rest (Fig. 9a, b, c, d). At present, she works as a part-time nurse in an adapted working scheme. She delivered three children and is coping reasonably well with her situation. The AOFAS hindfoot score was 65 at one and two years, 68 at three years, 72 at six years 60 at fifteen years (AOFAS, ankle-hindfoot score; 100points; excellent 90-100, good 75-89, failure < 50).

\section{DISCUSSION}

Total dislocation of talus from all its surrounding joints (tibiotalar, subtalar and talonavicular) is an extremely rare injury. Because of its rarity, only few case reports can be found in the literature. In the review of the literature, infection and AVN are the most commonly encountered complications that affect the outcome of these severe injuries. However, the high rate of complications has led may authors to suggest that tibio-calcaneal arthrodesis should be performed. This procedure often produce unwanted effects on the foot, particularly in young patients, because of loss of height and restriction of function of the peritalar joints. Management of totally extruded talus by reimplantation is although possible, but carries the risk of significant sepsis and even eventual loss of limb [5-7]. Recently, a paper was published by Magnan et al. Where they proposed to treat these injuries with a total body prosthesis and total ankle arthroplasty. They described the case of a forty-five years old male with an open enucleation of the talus treated in this way. After twenty eight months his ankle-hindfoot score, according to the American Orthopaedic Foot and Ankle Society was 92 of 100 points.

Herein, we report two cases of open total talar dislocation. Following a step-by-step algorithm, in the first step urgent radical debridement was done, with intravenously administrated antibiotics. To maintain the length of the hindfoot on one side and the midfoot on the other, the bone defect was filled with PMMA chains and external fixation, immobilizing the ankle and foot. In both cases a free flap was performed within the first ten days. Upon removal of the stitches the patients were restricted from bearing weight until transplantation. After three months, a fresh gender matched complete homologous talus was transplanted. The delay between the procurement of the talus and the actual implantation of it was 12 hours in the first and 8 hours in the second case.

Donors were located by the local multiple organ retrieval and exchange program. Graft procurement was carried out within 24 hours of death, under strict asepsis, with the specimen consisting of the entire joint with the capsule intact. After taking appropriate cultures, the graft was stored in one liter of Ringer's lactate at $4^{\circ} \mathrm{C}$ with added gentamycin $80 \mathrm{mg}$. 


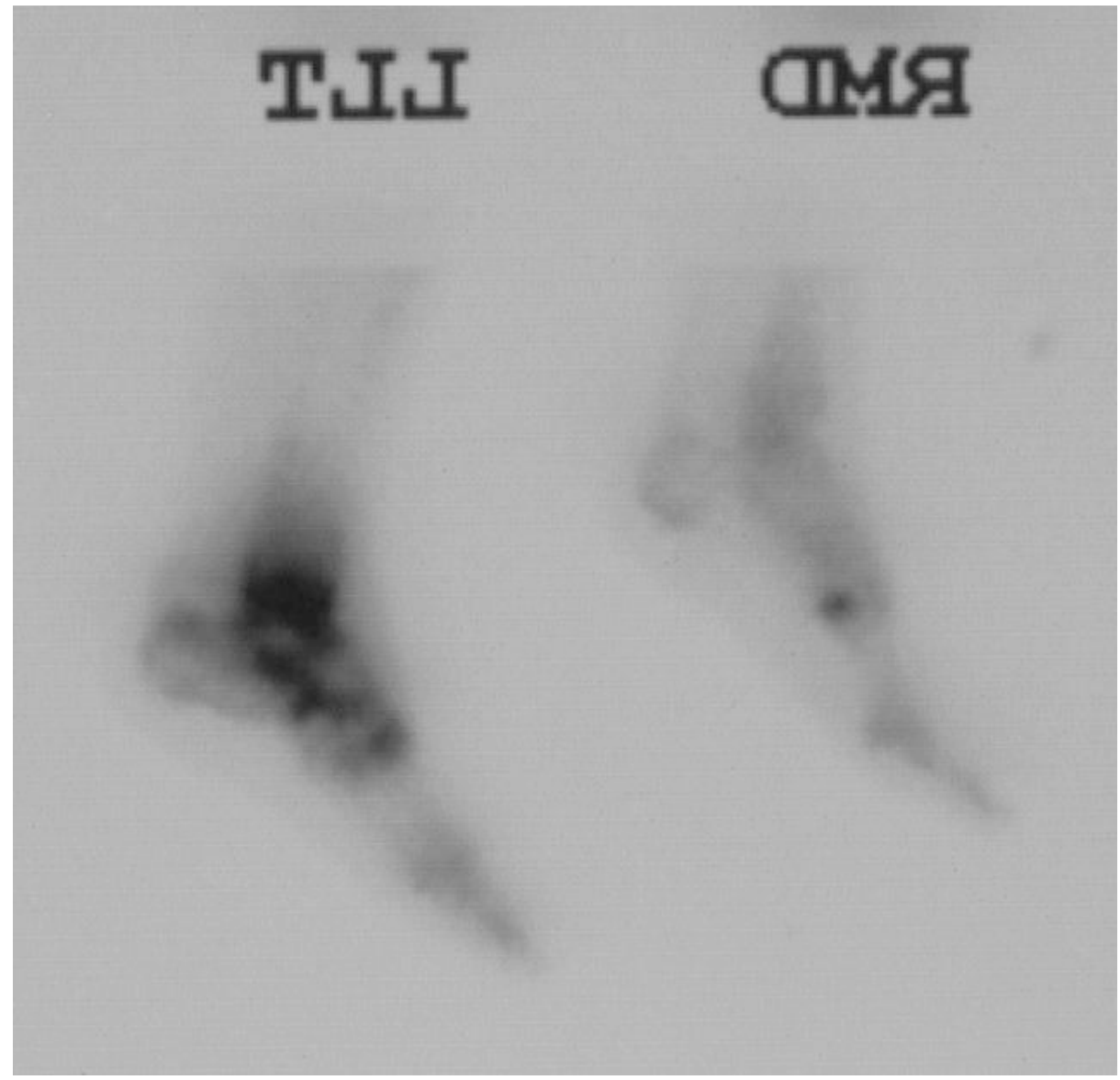

Fig. (10). Technetium scintigraphy of the ankle and foot of case I, 10 years after transplantation.

The rationale for utilizing fresh rather than preserved osteochondral allografts is that cartilage harvested without blood supply within 24 hours of the death of the donor is $100 \%$ viable and can be preserved for up to four days at $4^{\circ} \mathrm{C}$ [6].

Our result in the first case was poor, with a partial collapse of the transplanted talus and poor functional outcome. This collapse was not observed in our second case, and it scored on the ankle-hindfoot score of the AOFAS much better than the first case. We speculated that this is because in the first case there was a serious problem with sizing the graft to the ankle mortise. A middle segment of the transplanted talus had to be removed. Also, the inherent bony stability of the graft in the ankle mortise was poor; so additional ligamentous reconstruction of the lateral ankle bands with a strip of the peroneus brevis tendon had to be carried out.

The rational to use a massive osteochondral allograft to replace the lost talus, was to prevent considerable shortening of the leg and to preserve the maximum possible functional capability of the hind foot. In the first case we failed to obtain this goal, probably because of the problem of sizing the graft and lack of stability of the ankle joint. In this case an important collapse of the allograft was observed at the end of our follow-up. In the second case, the outcome was better as till now this patient can function with bearable discomfort, because the fitting of the graft was much better. In both cases no infections were observed. Remarkable is the restoration of vascularity of the allograft as was shown with the bone scan, six years after transplantation was remarkable.

Our conclusion of these cases is that massive fresh allografting of a total talus is possible with a reasonable outcome in young patients with traumatic loss of a complete talus, when precise fitting of the graft in ankle mortise of the host is possible. A stable tibiotalar joint seems to be essential for survival of the graft.

\section{CONFLICT OF INTEREST}

The authors confirm that this article content has no conflicts of interest.

\section{ACKNOWLEDGEMENTS}

Declared none.

\section{REFERENCES}

[1] Jaffe KA, Conlan TK, Sardis L, Meyer RD. Traumatic talectomy without fracture: four case reports and review of the literature. Foot Ankle Int. 1995; 16: 583-7. 
[2] Schuind F, Andrianne Y, Burny F, Donkerwolcke M, Saric O. Fractures et luxations de l'astragale. Acta Orthop Belg 1983; 49: 652-89.

[3] Brewster NT, Maffulli N. Reimplantation of the extruded talus. J Orthop Trauma 1997; 11: 42-5.

[4] Marsh JL,Saltzman CL,Iverson M, Shapiro DS. Major open injuries of the talus. J Orthop Trauma 1995; 9: 371-6.
[5] Palomo-Traver JM, Cruz-Renovell E, Granell-Beltran, MonzonisGracia J. Open total talus dislocation: case report and review of the literature. J Ortop Trauma 1997; 11: 45-9.

[6] Andrei A, Allan Gross E. Allografting in Orthopaedic Practice Williams \& Wilkins: Baltimore. 1992; pp. 67-82.

[7] Vaienti 1. Therapeutic management of complicated talar extrusion; literature review and case report. J Orthop Traumatol 2011 Mar; 12(1):61-4. doi: 10.1007/s10195-011-0129-z. [Epub 2011 Feb 25].

Received: September 03, 2014

Revised: December 01, 2014

Accepted: December 02, 2014

(c) Piet and Cristina; Licensee Bentham Open.

This is an open access article licensed under the terms of the Creative Commons Attribution Non-Commercial License (http://creativecommons.org/licenses/bync/3.0/), which permits unrestricted, non-commercial use, distribution and reproduction in any medium, provided the work is properly cited. 